ARTICLE

https://doi.org/10.1038/s41467-020-15544-x

\title{
Single-atom-layer traps in a solid electrolyte for lithium batteries
}

\author{
Feng Zhu',6, Md Shafiqul Islam²,6, Lin Zhou (i) ${ }^{3,6}$, Zhenqi Gu1, Ting Liu', Xinchao Wang', Jun Luo (i) ${ }^{5}$, \\ Ce-Wen Nan ${ }^{4}$, Yifei Mo (i) ${ }^{2 凶} \&$ Cheng Ma (10) ${ }^{1 凶}$
}

In order to fully understand the lithium-ion transport mechanism in solid electrolytes for batteries, not only the periodic lattice but also the non-periodic features that disrupt the ideal periodicity must be comprehensively studied. At present only a limited number of nonperiodic features such as point defects and grain boundaries are considered in mechanistic studies. Here, we discover an additional type of non-periodic feature that significantly influences ionic transport; this feature is termed a "single-atom-layer trap" (SALT). In a prototype solid electrolyte $\mathrm{Li}_{0.33} \mathrm{La}_{0.56} \mathrm{TiO}_{3}$, the single-atom-layer defects that form closed loops, i.e., SALTs, are found ubiquitous by atomic-resolution electron microscopy. According to ab initio calculations, these defect loops prevent large volumes of materials from participating in ionic transport, and thus severely degrade the total conductivity. This discovery points out the urgency of thoroughly investigating different types of non-periodic features, and motivates similar studies for other solid electrolytes.

\footnotetext{
${ }^{1}$ Division of Nanomaterials \& Chemistry, Hefei National Laboratory for Physical Sciences at the Microscale, CAS Key Laboratory of Materials for Energy Conversion, Department of Materials Science and Engineering, University of Science and Technology of China, Hefei, Anhui 230026, China. ${ }^{2}$ Department of Materials Science and Engineering, University of Maryland, College Park, MD 20742, USA. ${ }^{3}$ Ames Laboratory, U.S. Department of Energy, Ames, IA 50011, USA. ${ }^{4}$ School of Materials Science and Engineering, State Key Laboratory of New Ceramics and Fine Processing, Tsinghua University, Beijing 100084, China. ${ }^{5}$ Center for Electron Microscopy and Tianjin Key Lab of Advanced Functional Porous Materials, Institute for New Energy Materials \& Low-Carbon Technologies, School of Materials Science and Engineering, Tianjin University of Technology, Tianjin 300384, China. ${ }^{6}$ These authors contributed equally:

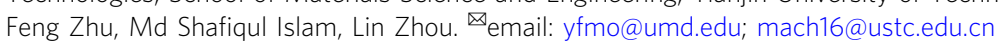


C ompared to the flammable organic liquid electrolyte in current commercial Li-ion batteries, solid electrolytes can greatly alleviate the safety issues, and also allow for further increase in energy density ${ }^{1-4}$. However, developing highly conductive solid electrolytes with comparable ionic conductivities to liquid electrolytes has been a grand challenge ${ }^{3-5}$. In order to effectively achieve this goal, a large body of research has been dedicated to studying the ionic transport mechanisms of solid electrolytes $^{3-5}$.

Fully comprehending the Li-ion transport mechanism in solid electrolytes requires in-depth studies not only to the periodic lattice, but also to structural features that deviate from the overall periodicity (referred to as "non-periodic features" below) $)^{3,4,6,7}$, such as point defects and grain boundaries. Since the existence of perfect, defect-free single crystals is forbidden by thermodynamics $^{8,9}$, solid electrolytes, like most materials, inevitably possess many non-periodic features ${ }^{7,9,10}$, and their impact on ionic transport is known to be crucial ${ }^{7,11-13}$. For example, grain boundaries can substantially lower the total ionic conductivity (frequently by orders of magnitude) in many solid electrolytes, including $\mathrm{Li}_{3} \mathrm{OX}(\mathrm{X}=\mathrm{Cl}$ or $\mathrm{Br})$ anti-perovskites ${ }^{14}, \mathrm{Li}_{7} \mathrm{La}_{3} \mathrm{Zr}_{2} \mathrm{O}_{12^{-}}$ based garnets ${ }^{15,16}, \mathrm{Li}_{0.33} \mathrm{La}_{0.56} \mathrm{TiO}_{3}$ (LLTO)-based perovskites ${ }^{6}$, and NASICON-type superionic conductors ${ }^{4,6}$. The introduction of interstitial $\mathrm{Li}^{+}$as point defects may often trigger the cooperative knock-on like mechanism ${ }^{17,18}$ and greatly enhance the conductivity, as exemplified by $\mathrm{Li}_{4} \mathrm{SiO}_{4}-\mathrm{Li}_{3} \mathrm{PO}_{4}{ }^{17}$, cubic $\mathrm{Li}_{7} \mathrm{La}_{3} \mathrm{Zr}_{2} \mathrm{O}_{12}{ }^{19,20}, \mathrm{Li}_{1+x} \mathrm{Al}_{x} \mathrm{Ti}_{2-x}\left(\mathrm{PO}_{4}\right)_{3}{ }^{21}$, and $\mathrm{LiTaSiO}_{5}{ }^{21,22}$. The $\mathrm{H}_{\mathrm{Li}}$ substitutional defects caused by hydration were found to strongly affect Li-ion migration in $\mathrm{Li}_{3} \mathrm{OCl}^{23}$ and Al-doped $\mathrm{Li}_{7} \mathrm{La}_{3} \mathrm{Zr}_{2} \mathrm{O}_{12}{ }^{24}$. The lithium-halide Schottky defect pairs may greatly facilitate Li-ion transport in multiple Li-rich antiperovskites ${ }^{23,25}$. Even the non-periodic features not directly involving charge carriers can significantly affect the ionic conductivity through the mixed polyanion effect ${ }^{26}$ and the plastic crystal behaviors like rotational motions of polyanions ${ }^{27}$. Clearly, before the Li-ion transport mechanism can be properly established, the non-periodic features must be thoroughly investigated first.

However, presently the understanding on the non-periodic features in Li-ion conductors is still limited. First of all, for many non-periodic features the atomistic mechanism controlling Li-ion migration is not well comprehended. A typical example is the grain boundaries. The studies that directly probed the atomicscale mechanism of grain-boundary Li-ion transport are limited, and most of them were reported only recently $14,15,28-30$. Dawson et al. directly simulated the grain boundaries in $\mathrm{Li}_{3} \mathrm{OCl}$ to clarify their role in ionic transport, and successfully reconciled the discrepancies among previous experimental and computational studies $^{14}$. Using a novel microscale strategy for simulation, they also elucidated the different contributions of grain-boundary resistance in sulfide and oxide solid electrolytes ${ }^{28}$. Additionally, the computational study of $\mathrm{Yu}$ et al. discovered that certain types of grain boundaries in $\mathrm{Li}_{7} \mathrm{La}_{3} \mathrm{Zr}_{2} \mathrm{O}_{12}$ may even facilitate ionic transport, and strategies for optimization was proposed accordingly ${ }^{15}$. These recent works provided valuable insights into both the diffusion mechanisms and conductivity improvement, but similar investigations remain scarce in literature. In order to comprehensively understand the influence of non-periodic features, more of such in-depth studies are needed. Secondly, so far only a few types of non-periodic features like point defects and grain boundaries have received attention. Nevertheless, many other non-periodic features, e.g., dislocations, stacking faults, and twin boundaries, could also be intrinsically existent in solid electrolytes ${ }^{9,31}$, and some of them might significantly influence the Li-ion migration too. Before the ionic transport mechanism can be fully understood, identifying these relevant non-periodic features and thoroughly understanding their influence on Li-ion migration would be indispensable. Regardless, the highly localized nature and low volume fraction of most non-periodic features ${ }^{9}$ make such exploration quite challenging ${ }^{3,6,7}$. Presently, the nonperiodic features involved in most mechanistic studies are still limited to a few types like point defects and grain boundaries ${ }^{3,4}$.

Here, we discover an additional type of non-periodic feature that significantly influences Li-ion migration, and its role in ionic transport is unravelled at the atomic scale by combining advanced electron microscopy and $\mathrm{ab}$ initio calculations. In a prototype solid electrolyte $\mathrm{Li}_{0.33} \mathrm{La}_{0.56} \mathrm{TiO}_{3}$ (LLTO) ${ }^{3,32}$, closed loops formed by a kind of single-atom-layer $2 \mathrm{D}$ defect are found ubiquitous by electron microscopy observation; a term "single-atom-layer trap" (SALT) is coined to describe such defect loops. Based on the experimentally determined defect structure, ab initio calculations unambiguously demonstrate that the SALTs, although never discussed in previous mechanistic studies, prevent large volumes of materials from participating in ionic transport, and severely degrade the total conductivity.

\section{Results}

Spotting SALTs in the lattice. In order to investigate the nonperiodic features that are relevant to ionic transport, a prototype solid electrolyte, $\mathrm{Li}_{0.33} \mathrm{La}_{0.56} \mathrm{TiO}_{3}$ (LLTO), was selected for study. Crystallizing in the perovskite structure, it shows a very high bulk conductivity of $10^{-3} \mathrm{~S} \mathrm{~cm}^{-1}$, approaching that of liquid electrolytes $\left(10^{-2} \mathrm{~S} \mathrm{~cm}^{-1}\right)^{32,33}$. The LLTO ceramics used in the present work were prepared by the common sintering method ${ }^{7}$. The inductively coupled plasma (ICP) spectroscopy suggested that the molar ratio of Li:La:Ti was $0.325: 0.552: 1$, nearly identical to the nominal stoichiometry $0.33: 0.56: 1$. The $\mathrm{x}$-ray diffraction pattern (Supplementary Fig. 1) suggested that the ceramics were phasepure with the tetragonal perovskite structure, consistent with those prepared under the same conditions ${ }^{7,32}$. Besides, the ionic conductivities measured by both the AC (Supplementary Figs. 2 and 3$)$ and DC methods $\left(3.55 \times 10^{-5} \mathrm{~S} \mathrm{~cm}^{-1}\right)$ also agreed very well with the reported values in literature ${ }^{32-35}$. However, atomicresolution scanning transmission electron microscopy (STEM) observation spotted a large population of single-atom-layer 2D defects that had never been considered in previous mechanistic studies. Figure la shows a representative high-angle annular dark-field (HAADF) STEM image of such defects. Within the lattice of LLTO (regions with brighter contrast), dark straight lines were clearly visible. Given that STEM images are essentially the $2 \mathrm{D}$ projection of $3 \mathrm{D}$ objects ${ }^{36}$, the defects corresponding to these dark lines were actually atomic planes parallel with the observing direction, i.e., $\langle 100\rangle_{\mathrm{p}}$ of LLTO (the subscript $\mathrm{p}$ refers to the prototype perovskite unit cell). Since the image intensity of HAADF-STEM reflects the average atomic number ${ }^{36}$, the observed dark planes must exhibit a very different composition from LLTO. It should be noted that these defects were not caused by the specimen thinning procedure that is required for highquality STEM imaging. Supplementary Fig. 4 shows the TEM images of "pristine" LLTO particles, which were obtained by manually crushing the sintered LLTO ceramic and did not undergo any thinning procedure. Despite the low image quality caused by the large specimen thickness, the defects were still spotted for multiple times, indicating that they are intrinsically existent within the material. Although these defects had never been investigated in detail before, they were surprisingly easy to find. A few more examples are displayed in Fig. 1b-d. After examining a large number of them, we discovered three important characteristics. First of all, they may only lie within one of the $\{001\}_{\mathrm{p}}$ planes of LLTO (Fig. 1a-d). As a result, the interconnected $2 \mathrm{D}$ defects were always perpendicular to each other. Secondly, the 
a

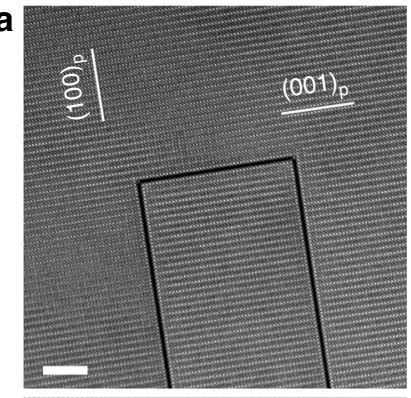

C

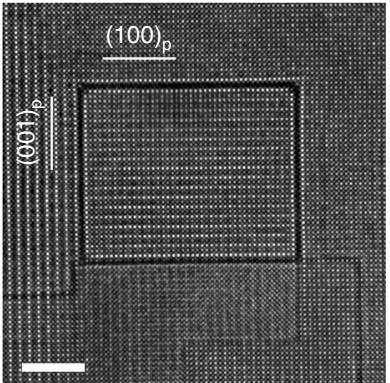

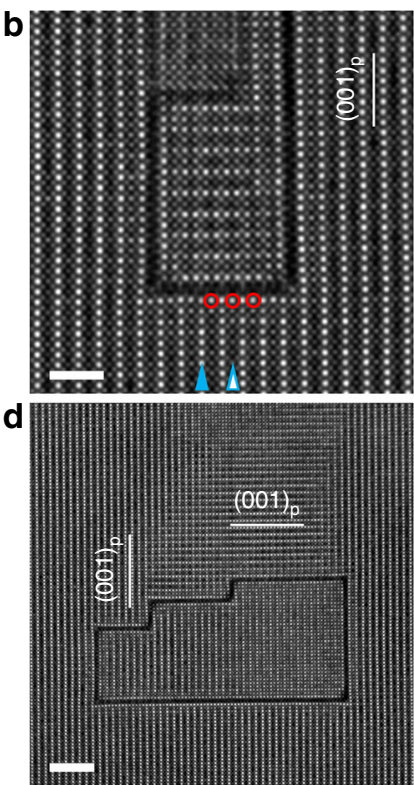

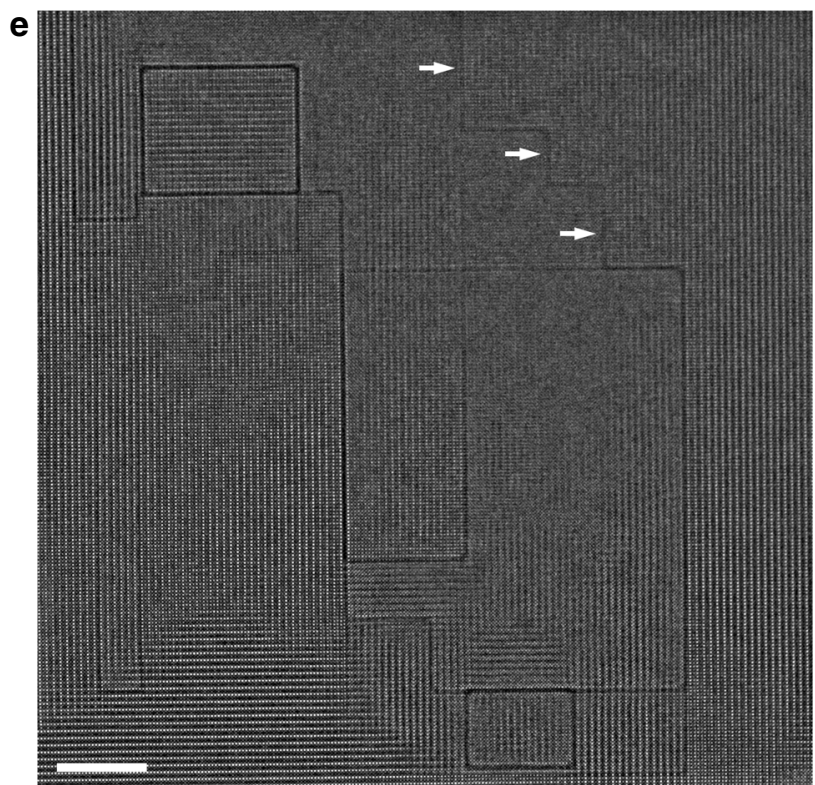

Fig. 1 Single-atom-layer traps (SALTs) in LLTO. a-d HAADF-STEM images of the 2D defects that formed SALTs. The LLTO planes parallel with the 2D defects are marked directly in images. A La-rich A-site layer and a La-poor one in LLTO were indicated by solid and empty arrows, respectively, in b. The red circles indicated three A-site atomic columns that were located in the La-poor layer of LLTO but became La-rich because of the neighboring $2 \mathrm{D}$ defect layer. e Ubiquitous presence of SALTs. The bright arrows indicated three 2D defects that appeared less dark due to the coexisting LLTO in their atomic planes. The scale bars in $\mathbf{a}, \mathbf{b}, \mathbf{c}, \mathbf{d}$ and $\mathbf{e}$ are $5 \mathrm{~nm}, 2 \mathrm{~nm}, 5 \mathrm{~nm}, 5 \mathrm{~nm}$ and $10 \mathrm{~nm}$, respectively.

presence of the $2 \mathrm{D}$ defect appeared to influence the composition of its nearest neighboring LLTO planes. LLTO is characterized by the alternate stacking between La-rich and La-poor A-site layers, which would appear as rows of bright and dark spots in the HAADF-STEM images, respectively ${ }^{7}$; in Fig. $1 \mathrm{~b}$, a La-rich layer and a La-poor one were pointed by solid and empty arrows, respectively. Regardless, the A-sites closest to the defect always showed brightness (and thus composition) nearly identical to that of La-rich ones in bulk LLTO, even if some of them were located in the La-poor layer and should have been dark. In Fig. 1b, a few examples of such A-sites were circled in red. Last but not least, most (if not all) of the defects formed "closed loops", isolating a volume of LLTO from the rest of the grain. Actually, besides the defects in Fig. 1c and d, those in Fig. 1a, b also formed closed loops, but they were too big to be shown completely at high magnifications. Such loops were so ubiquitous that they even shared edges with each other from time to time (Fig. 1e). Among the defects constituting these loops, some were coexisting with LLTO in the defect plane, and thus appeared less dark due to the brighter contrast of LLTO (a few examples pointed by bright arrows in Fig. 1e). In addition to the two $\{001\}_{p}$ planes parallel with the viewing direction $\langle 100\rangle_{\mathrm{p}}$ in Fig. 1 , the third $\{001\}_{\mathrm{p}}$ that coincided with the viewing plane could also contain $2 \mathrm{D}$ defects: after all, such features can be parallel with any of the three $\{001\}_{\mathrm{p}}$ planes (Fig. 1a-d). Among these three differently oriented 2D defects, any two could and would almost always form closed loops with each other (in fact, the "stand-alone" 2D defect that did not form closed loops with others was barely observed). Consequently, although only the defects in two of the three $\{001\}_{p}$ planes can appear edge-on and be visible along the viewing direction $\langle 100\rangle_{\mathrm{p}}$ in Fig. 1, it is highly likely that defects also existed in the third $\{001\}_{\mathrm{p}}$ plane, i.e., the viewing plane, to form closed loops with the ones appearing edge-on in the images. That is, the $2 \mathrm{D}$ defects in all the three $\{001\}_{\mathrm{p}}$ planes would often (if not always) enclose $3 \mathrm{D}$ volumes together by forming the observed loops. It should be noted that the $2 \mathrm{D}$ defects here may not be considered as a special case of grain boundaries, as the loops formed by them actually existed within individual grains (Supplementary Fig. 5). Besides, as shown in Supplementary Fig. 5a, grain boundaries lacked many of the aforementioned characteristics for $2 \mathrm{D}$ defects, e.g., lying exclusively in $\{001\}_{\mathrm{p}}$, being visible only along certain orientations, and always forming right angle with each other. Therefore, although the observed defect may enclose 3D volumes like grain boundaries, it is a fundamentally different non-periodic feature. We coined a term called "singleatom-layer trap" (SALT) to describe the closed loops formed by these defects. Clearly, the atomic framework for Li-ion migration, as shown in Fig. 1e, cannot be fully described without taking SALTs into account.

Composition and structure of the 2D defects in SALTs. Before the role of SALTs in ionic transport can be studied, the composition and structure of the $2 \mathrm{D}$ defects constituting them must be unraveled first. To start with, the energy-dispersive X-ray spectroscopy (EDX) was performed on a SALT shown in Fig. 2a. In the mapping result of La (Fig. $2 \mathrm{~b}$ ), a dark loop with exactly the same geometry as the SALT was immediately revealed, indicating that the La content was negligibly small. This is consistent with the HAADF-STEM imaging: considering that $\mathrm{La}$ is the heaviest element in LLTO and that HAADF-STEM reflects average atomic numbers ${ }^{36}$, the absence of La in SALTs should naturally lead to dark image contrast. Beyond La, the variation of other elements was studied by electron energy loss spectroscopy (EELS), which is more sensitive to light elements ${ }^{37,38}$. In the region shown in Fig. $2 c$, the Li- $K$ signal was collected in an EELS line scan running across two adjacent $2 \mathrm{D}$ defects. In order to confirm that the $\sim 62$ $\mathrm{eV}$ signal acquired during the line scan is indeed $\mathrm{Li}-K$ rather than Ti- $M_{1}{ }^{39}$, EELS was performed to the La-rich (Li-poor) and Lapoor (Li-rich) layers of LLTO (Supplementary Fig. 6a), which differed greatly in $\mathrm{Li}$ content but exhibited the same $\mathrm{Ti}$ content ${ }^{7,34,40}$. As shown in Supplementary Fig. $6 \mathrm{~b}$, the $62 \mathrm{eV}$ signal was found much stronger in the Li-rich layer, instead of showing similar intensities in both layers like the Ti content. 

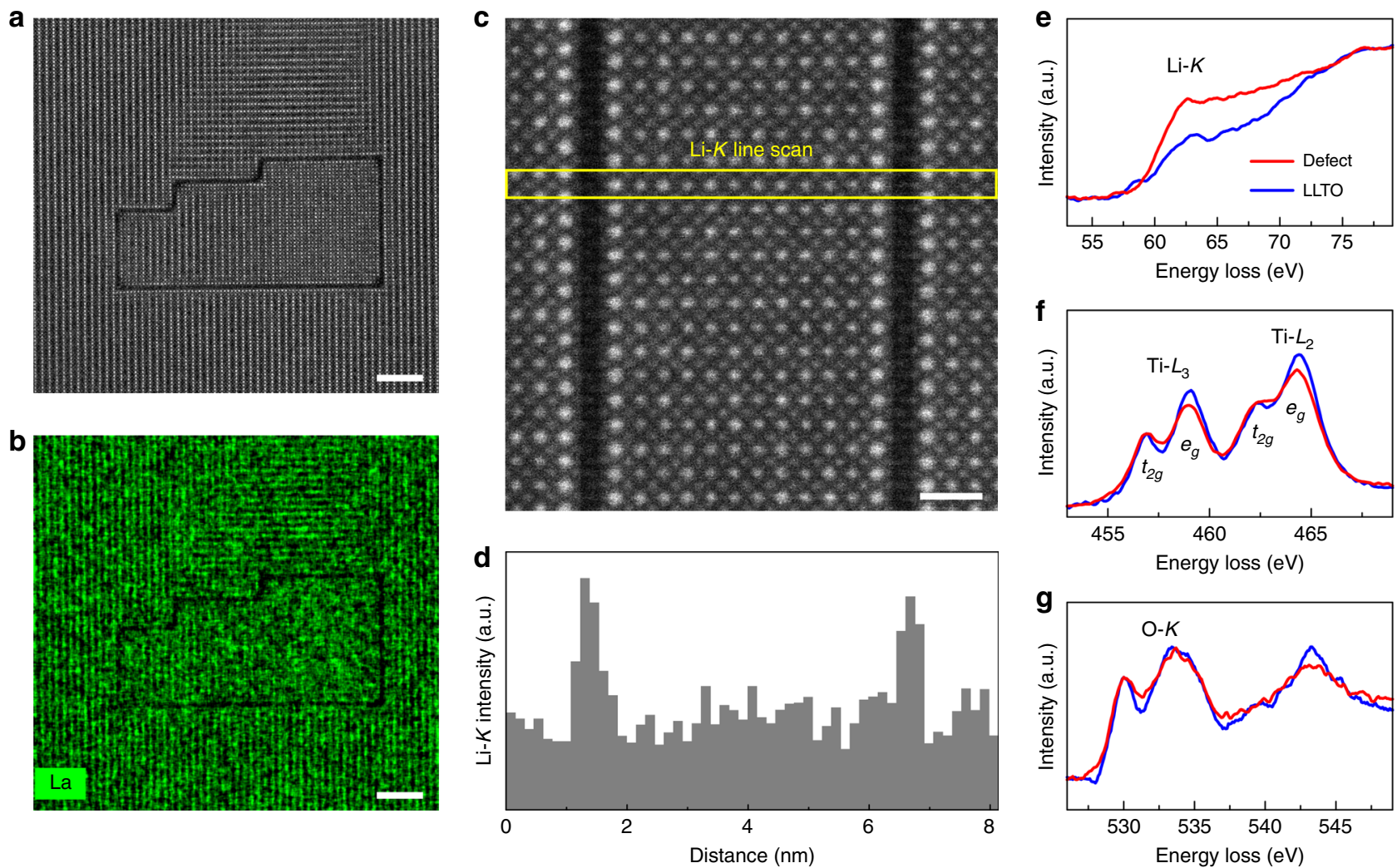

Fig. 2 EDX and EELS analyses of the 2D defects. a, b HAADF-STEM image (a) and the corresponding EDX mapping result (b) of a SALT formed by the 2D defects. The scale bars in $\mathbf{a}$ and $\mathbf{b}$ are both $5 \mathrm{~nm}$. c, $\mathbf{d}$ HAADF-STEM image (c) and the Li- $K$ intensity profile (d) obtained via EELS line scan in the region indicated by the yellow rectangle in $\mathbf{c}$. The scale bar in $\mathbf{c}$ is $1 \mathrm{~nm}$. e-g EELS results of Li-K (e), Ti- $L_{2,3}(\mathbf{f})$, and O-K ( $\left.\mathbf{g}\right)$ for the $2 \mathrm{D}$ defect and bulk LLTO.

Consequently, the $62 \mathrm{eV}$ signal can be safely designated as Li- $K$. In the EELS line scan result (Fig. 2d), the integrated intensity of Li- $K$ clearly peaked at both defects, indicating an enrichment of Li. In addition to the content, the electronic environment of $\mathrm{Li}$ was also quite dissimilar between LLTO and the defect, as reflected by their different $\mathrm{Li}-\mathrm{K}$ fine structures (Fig. $2 \mathrm{e}$ ) ${ }^{37}$. As for $\mathrm{Ti}$ and $\mathrm{O}$, no significant content fluctuation was observed across the defects (Fig. 2f-g), but the EELS fine structures disclosed interesting information. The Ti- $L_{2,3}$ white lines are sensitive to the oxidation state of $\mathrm{Ti}$, whose variation would be reflected in the $L_{2} / L_{3}$ intensity ratio and peak positions ${ }^{41,42}$. Figure $2 \mathrm{f}$ suggested that the defects and LLTO were virtually the same in both aspects. As such, the valence of Ti should remain $4+$ within the defects. Additionally, the $t_{2 g} / e_{g}$ peak splitting of Ti- $L_{2,3}$ in LLTO also existed in the defect. This splitting arose from the bonding of $\mathrm{Ti}$ with its six neighboring $\mathrm{O}^{6,42}$, so most likely the $\mathrm{TiO}_{6}$ octahedra similar to those in LLTO were present in the defects too. However, the octahedra in the latter should be distorted in a slightly different way from the former, as their $t_{2 g} / e_{g}$ intensity ratios were not exactly the same ${ }^{42}$. Finally, the comparison of $\mathrm{O}-\mathrm{K}$ fine structures suggested that the bonding of $\mathrm{O}$ with adjacent cations within defects should also be distinct from that in LLTO $^{37}$. According to the EDX and EELS analyses presented above, the $2 \mathrm{D}$ defect should essentially be a single-atom-layer Li-Ti-O compound that was Li-rich and possessed $\mathrm{TiO}_{6}$ octahedra with $\mathrm{Ti}^{4+}$.

Based on these results, the structure of the defect was probed by atomic-resolution STEM observation. The study began with the configuration of LLTO unit cells at different sides of the defect. When LLTO was aligned along $[1 \overline{1} 0]_{p}$ (Fig. 3a), the perovskite unit cells at one side of the defect appeared to match with those at the other. That is, if the defect were replaced by an ordinary layer of LLTO, these unit cells would form a continuous, perfect lattice. Nevertheless, when LLTO was oriented along $[010]_{p}$ (Fig. 3b), a mismatch by half of the lattice parameter was observed. The difference between images along $[1 \overline{1} 0]_{p}$ and $[010]_{p}$ indicated that the LLTO unit cells at one side of the $2 \mathrm{D}$ defect must be shifted with respect to those at the other by $1 / 2[\overline{1} 10]_{p}$, as shown in Supplementary Fig. 7a. In this way, when multiple unit cells were present, projecting the entire $3 \mathrm{D}$ object to the viewing planes in Fig. 3a, b, as shown in Supplementary Fig. 7b, c, respectively, would precisely produce the appearances described above. With the configuration of neighboring LLTO unit cells determined, the structure of the 2D defect itself may be analyzed. It was found that, no matter whether the observation was performed along $[1 \overline{1} 0]_{p}$ (Fig. 3a, c) or $[010]_{p}$ (Fig. 3b, d) of LLTO, the defect always exhibited well defined, highly ordered atomic columns like those in a periodic lattice. Therefore, albeit singleatom-layer thick, it may very likely be isostructural with a known compound that was capable of forming epitaxy with perovskites. Combining this clue with the characteristics learnt from Fig. 2, i.e., $\mathrm{Li}-\mathrm{Ti}-\mathrm{O}$ compound, $\mathrm{TiO}_{6}$ octahedra, $\mathrm{Ti}^{4+}$, etc., the rock-saltstructured $\mathrm{Li}_{2} \mathrm{TiO}_{3}$ with $F m \overline{3} m$ space group ${ }^{43}$, i.e., $\gamma-\mathrm{Li}_{2} \mathrm{TiO}_{3}$, immediately arose as a highly probable candidate. It is well known that the rock-salt structure can easily interface with perovskites in an epitaxial manner ${ }^{44,45}$. Besides, the rock-salt $\gamma$ $\mathrm{Li}_{2} \mathrm{TiO}_{3}$ did exhibit $\mathrm{TiO}_{6}$ octahedra, and the Ti valence was also $4+$. If it interfaced epitaxially with LLTO, the $<110>$ and $<010\rangle$ would be parallel with $\langle 110\rangle_{\mathrm{p}}$ and $\langle 010\rangle_{\mathrm{p}}$ of LLTO, respectively, according to the reported epitaxial relationship ${ }^{44}$. Therefore, should the 2D defect observed here be a single-atom-layer $\gamma$ $\mathrm{Li}_{2} \mathrm{TiO}_{3}$ and follow such orientations, it may only be the atomic plane containing both $<110>$ and $<010>$, i.e., the $\{001\}$ plane. In 

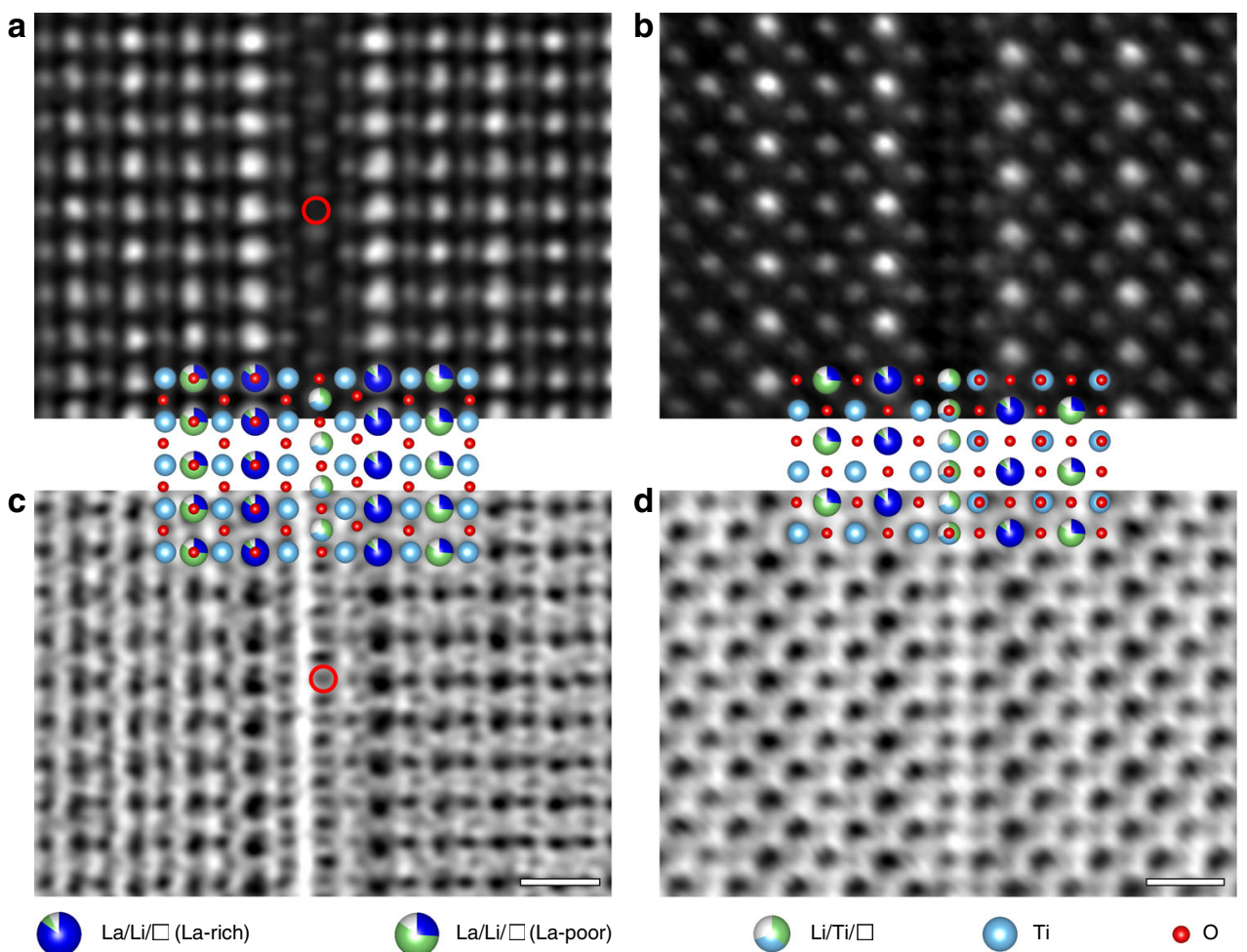

Fig. 3 Structural analysis of the 2D defects. a, c HAADF-STEM (a) and ABF-STEM (c) images of the defect when the adjacent LLTO was oriented along $[110]_{p}$. An atomic column consisting of $\mathrm{O}$ only was circled in red in $\mathbf{a}$ and $\mathbf{c}$. $\mathbf{b}, \mathbf{d}$ HAADF-STEM (b) and ABF-STEM (d) images of the defect when the adjacent LLTO was oriented along $[010]_{p}$. The scale bars in $\mathbf{c}$ and $\mathbf{d}$ are both $5 \AA$.

order to verify whether the defect really took this structure, we constructed the atomic model accordingly and compared it with the STEM images in Fig. 3. The comparison was first made along the $[1 \overline{1} 0]_{p}$ zone axis of LLTO. When the LLTO unit cells in the atomic model were aligned with the HAADF-STEM image (Fig. 3a), the Li/ Ti columns of the defect matched very well with the bright spots. Since HAADF-STEM cannot easily detect light elements, the locations corresponding to pure $\mathrm{O}$ columns (one of them circled in red) in the atomic model barely showed any contrast. However, when examined by ABF-STEM (Fig. 3c), which is sensitive to elements with small atomic numbers ${ }^{46}$, the $\mathrm{O}$ columns were clearly visualized at expected positions too (one of which circled in red). In order to further confirm the speculated defect structure, we also made the comparison when LLTO was aligned along $[010]_{p}$. To this end, the entire atomic model, i.e., both the defect and the neighboring LLTO unit cells, in Fig. 3a, c was rotated around $[001]_{p}$ of LLTO by $135^{\circ}$, so that LLTO was tilted from $[1 \overline{1} 0]_{p}$ to $[010]_{p}$. Then, this re-oriented atomic model was compared with the HAADF- and ABF-STEM images taken along $[010]_{p}$ of LLTO (Fig. 3b, d, respectively). Again, they matched perfectly with each other. Therefore, it should be safe to conclude that the speculation above was correct: the $2 \mathrm{D}$ defect was indeed isostructural with the $\{001\}$ plane of the rock-salt-structured $\gamma-\mathrm{Li}_{2} \mathrm{TiO}_{3}$.

Nevertheless, exhibiting the rock-salt structure does not necessarily mean that the stoichiometry is precisely $\mathrm{Li}_{2} \mathrm{TiO}_{3}$. In fact, the composition of the $2 \mathrm{D}$ defect must ensure an overall charge balance. This was schematically illustrated in Supplementary Fig. 8. First of all, the unit cell of LLTO consists of one La-rich, one La-poor, and two Ti-O planes parallel with $(001)_{\mathrm{p}}$. Each Ti-O plane shows a Ti/O ratio of $1 / 2$, and is thus charge balanced. The compositions of La-rich and La-poor layers here cannot be determined precisely, but an acceptably good approximation can be found in a recent neutron diffraction study ${ }^{40}$, where the stoichiometry of the material under study (confirmed by ICP analysis) was also $\mathrm{Li}_{0.33} \mathrm{La}_{0.56} \mathrm{TiO}_{3}$. In this way, the La-rich and Lapoor layers within the unit cell shown in Supplementary Fig. 8 can be estimated to carry +0.62 and -0.62 charges, respectively, which set off each other to maintain the charge balance of the unit cell. Since the A-site layers neighboring the defect are nearly identical with the La-rich ones in bulk LLTO, as reflected by their similar intensities in the Z-contrast images (Fig. 1), the in-between 2D defect must exhibit the -0.62 charge that should have been carried by a La-poor layer. Such a negative charge entailed that the $2 \mathrm{D}$ defects, unlike the charge balanced $\gamma-\mathrm{Li}_{2} \mathrm{TiO}_{3}$, should contain cation vacancies. Assuming these vacancies arising from $\mathrm{Li}$ deficiency only, each Li/Ti/vacancy site in Fig. 3 and Supplementary Fig. 8 should contain $0.37 \mathrm{Li}, 0.33 \mathrm{Ti}$, and 0.30 vacancies to reach -0.62 charge within the "unit cell" of the defect plane (such a "unit cell", as shown in Supplementary Fig. 8, consisted of $2 \mathrm{Li} / \mathrm{Ti} /$ vacancy and $2 \mathrm{O}$ sites). In this configuration, the Li concentration within the defect layer may thus be calculated to be around 2.23 times of that in bulk LLTO. Interestingly, this was quite close to the value obtained by the quantification analysis of Li- $K$ edges in Fig. 2e (2.06), indicating the stoichiometry raised above, albeit a rough estimation, should not deviate much from reality. Additionally, we also simulated the STEM images using this atomic model, and the results (Supplementary Fig. 9) agreed well with the experimental observation in Fig. 3 too (the minor discrepancies arose from the distractions that are inevitable in experimental imaging but cannot be precisely incorporated in simulation, e.g., drifting, inaccuracy of specimen alignment, and uncertainty of the specimen thickness). Therefore, the $2 \mathrm{D}$ defect should be a single-atom-layer compound that is isostructural with $\{001\}$ of the rock-salt $\gamma-\mathrm{Li}_{2} \mathrm{TiO}_{3}$ and exhibits an estimated composition of $\left[\mathrm{Li}_{0.37} \mathrm{Ti}_{0.33} \mathrm{O}\right]^{0.31-}$ (or $\left[\mathrm{Li}_{1.11} \mathrm{TiO}_{3}\right]^{0.93-}$ ). The atomic configuration within the defect plane was shown schematically in Supplementary Fig. 10. 
In order to investigate how the $2 \mathrm{D}$ defect was connected with LLTO, geometric phase analysis (GPA) ${ }^{47}$ was performed. Under most circumstances, the epitaxial growth of one phase on the other would be accompanied by periodic misfit dislocations at the interfaces, so that their lattice mismatch can be reconciled ${ }^{48}$. Surprisingly, this phenomenon was not observed at the $2 \mathrm{D}$ defect, even if the lattice parameter of bulk $\gamma-\mathrm{Li}_{2} \mathrm{TiO}_{3}(4.10 \AA)$ is much larger than that of LLTO (3.87 $\AA$ ). According to the GPA results (Fig. 4), only $\varepsilon_{x x}$ maximized at the $2 \mathrm{D}$ defect, reflecting a local enlargement of the distance between atomic planes perpendicular

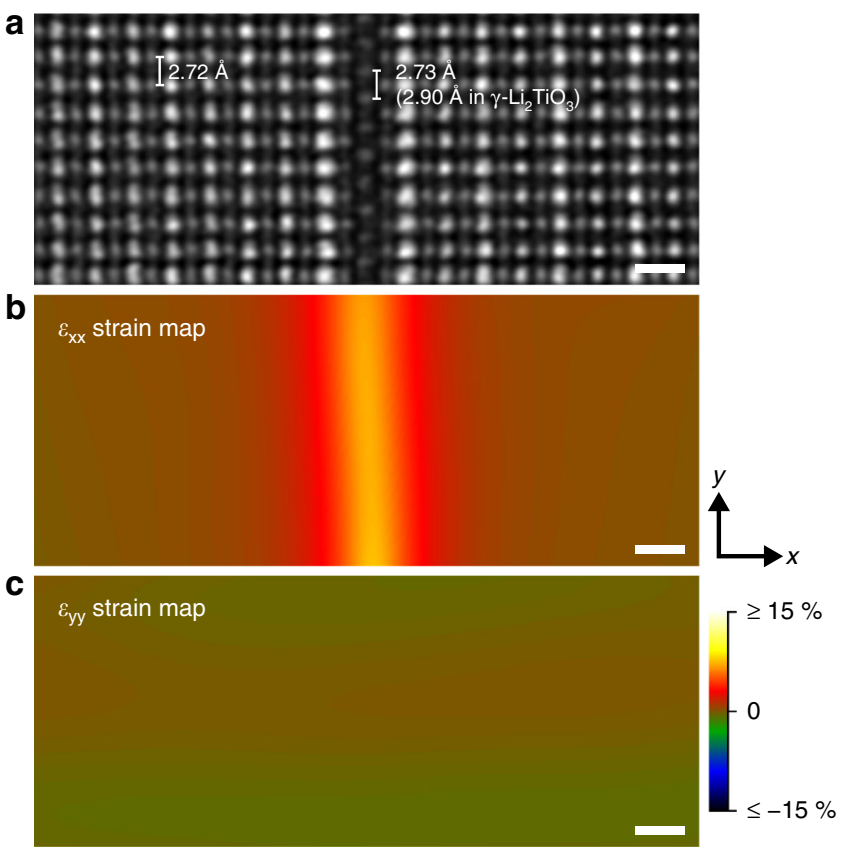

Fig. 4 Strain variation across the 2D defects. a HAADF-STEM image of a 2D defect with the adjacent LLTO oriented along $[110]_{p}$. b, c $\varepsilon_{\mathrm{xx}}(\mathbf{b})$ and $\varepsilon_{\mathrm{yy}}$ (c) strain maps obtained by performing GPA on the image in $\mathbf{a}$. The scale bars in a-c are all $5 \AA$. to the defined $x$ direction, while $\varepsilon_{y y}$ did not show any fluctuation that can indicate the existence of misfit dislocations. The interatomic distances were investigated to unravel the mechanism behind. It was found that the distance between two neighboring atomic columns of the $2 \mathrm{D}$ defect in Fig. 4 a was only $2.73 \AA$. This value was much smaller than the same spacing in bulk $\gamma-\mathrm{Li}_{2} \mathrm{TiO}_{3}$ ( $2.90 \AA)$ but matched quite well with that in LLTO $(2.72 \AA)$. The compactness of single-atom-layer $\left[\mathrm{Li}_{1.11} \mathrm{TiO}_{3}\right]^{0.93-}$ with respect to $\gamma-\mathrm{Li}_{2} \mathrm{TiO}_{3}$ may be attributed to its $\mathrm{Li}$ deficiency, or compression from the surrounding LLTO, or both. Regardless, thanks to such a contraction of interatomic distances, the $2 \mathrm{D}$ defects were able to fit smoothly into the LLTO lattice, without involving any dislocations.

SALTs versus Li-ion transport. In order to investigate the influence of SALTs on ionic transport, the interplay between individual 2D defects and Li-ion migration must be understood first. To this end, we constructed the atomic model for firstprinciples calculations based on the defect structure determined above. Since the LLTO lattice at one side of the defect was shifted by $1 / 2[\overline{1} 10]_{p}$ with respect to that at the other (Supplementary Fig. 7a), the individual LLTO/defect/LLTO sandwich does not satisfy the periodic boundary condition along the $[001]_{\mathrm{p}}$ direction of LLTO, and thus cannot be used directly for calculations. As a result, we constructed the atomic model via the back-to-back combination of two LLTO/defect/LLTO sandwiches instead. In such a model (Fig. 5a), the LLTO lattice on the right side was shifted by $1 / 2[\overline{1} 10]_{p}$ with respect to that in the middle, which was also shifted by $1 / 2[\overline{1} 10]_{p}$ with respect to that on the left side. Consequently, the LLTO lattice on the right side was shifted by $[\overline{1} 10]_{p}$ with respect to that on the left, satisfying the periodic boundary condition. With the atomic model constructed this way, the experimentally determined defect structure was confirmed by density functional theory (DFT) calculations.

$\mathrm{Ab}$ initio molecular dynamics (AIMD) simulations were then performed to investigate the Li-ion transport behavior. During the simulation, we observed no Li-ion diffusion crossing between the $2 \mathrm{D}$ defect layer and the neighboring LLTO. This can be a
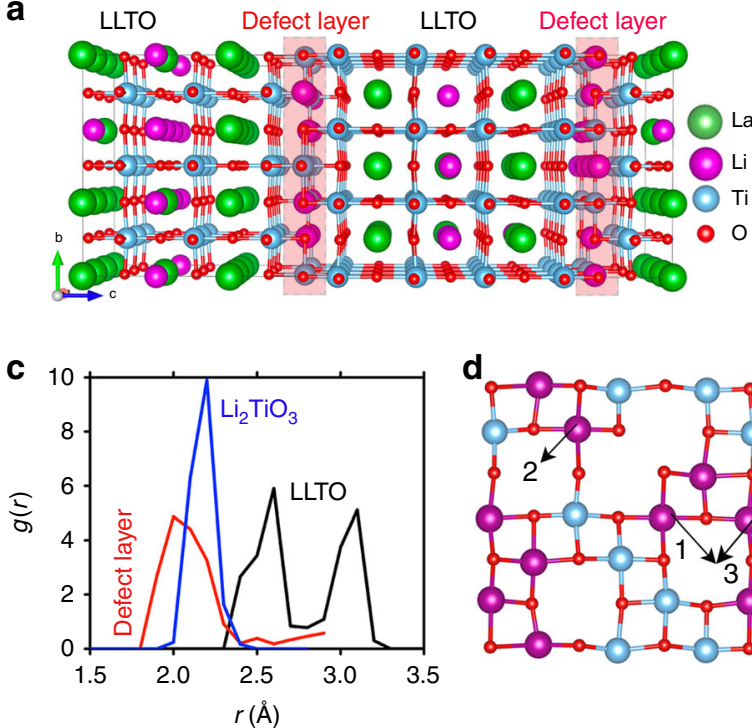
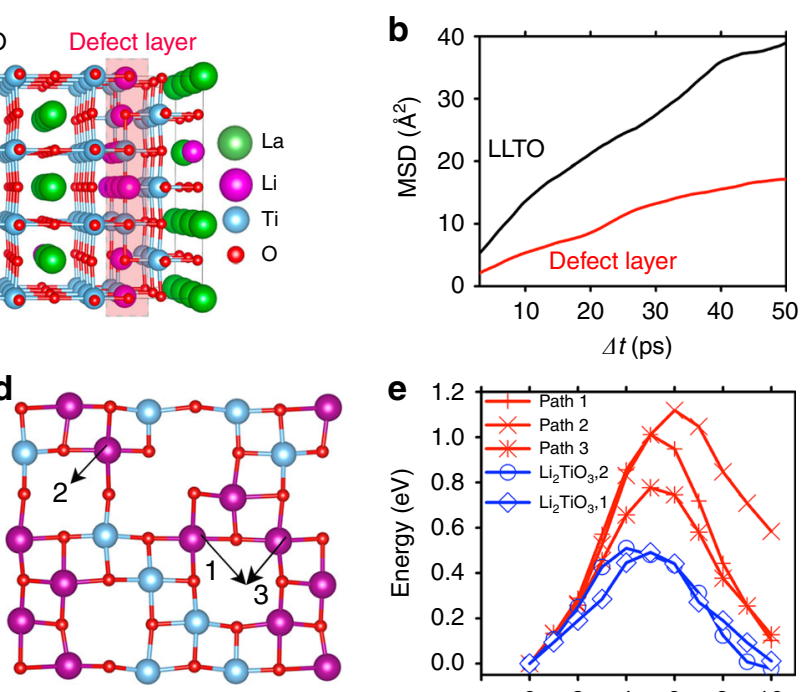

Fig. 5 First principles atomistic modeling of the 2D defects. a Atomistic model used for calculations. b MSD of Li ions in bulk LLTO versus that within (and also parallel with) the 2D defect layer from AIMD simulations at $2000 \mathrm{~K}$. c Radial distribution functions $g(r)$ of Li-O bonds in the $2 \mathrm{D}$ defect layer, $\beta$ $\mathrm{Li}_{2} \mathrm{TiO}_{3}$, and bulk LLTO. The 2D defect layer has shorter $\mathrm{Li}-\mathrm{O}$ bond length than bulk LLTO and $\beta-\mathrm{Li}_{2} \mathrm{TiO}_{3}$. $\mathbf{d}$ Li-ion migration pathways selected for NEB calculations in the 2D defect layer. e Migration energy profiles of selected Li-ion pathways in the 2D defect layer (red data, pathways indicated in $\mathbf{d}$ ) and of similar pathways within the (202) plane of $\beta-\mathrm{Li}_{2} \mathrm{TiO}_{3}$ (blue data, pathways indicated in Supplementary Fig. 12). 
explained by the La-rich LLTO layers adjacent to the 2D defect, which are known to be Li-ion-blocking due to the strong repulsion of $\mathrm{La}^{3+}$ and limited number of vacancies for $\mathrm{Li}$-ion hopping ${ }^{49,50}$. Because of these non-conductive atomic layers, Liion diffusion cannot happen in the perpendicular direction across the defect layer. In the directions parallel with the defect layer, the Li-ion diffusion within the 2D defect was found much slower than that in the bulk phase of LLTO, as measured by the Li-ion mean square displacement (MSD) over time (Fig. 5b). The poorer ionic transport may be attributed to the compressed volume for Li-ion migration, which resulted from the close packing of Li-O within the $2 \mathrm{D}$ defect. According to the calculated radial distribution functions $g(r)$ of $\mathrm{Li}-\mathrm{O}$, the distance between $\mathrm{Li}$ and $\mathrm{O}$ was much shorter in the $2 \mathrm{D}$ defect than in LLTO (Fig. $5 \mathrm{c}$ ). The resulting smaller volume increased the migration barrier for $\mathrm{Li}$ ions, and thus led to the poor mobility reflected in Fig. 5b. We further compared the $2 \mathrm{D}$ defect with $\beta-\mathrm{Li}_{2} \mathrm{TiO}_{3}$ (space group $C 2 / c)^{51}$. This compound shows similar atomic configurations with the 2D defect in its (202) plane, and the ionic conductivity is $\mathrm{known}^{51,52}$; based on the Arrhenius plot between 300 and $600{ }^{\circ} \mathrm{C}^{52}$, its room-temperature conductivity can be estimated as $2.43 \times 10^{-12} \mathrm{~S} \mathrm{~cm}^{-1}$. Compared to such a poor ionic conductor, the $2 \mathrm{D}$ defect, with shorter Li-O distances (Fig. $5 \mathrm{c}$ ), shows even slower Li-ion diffusion, as confirmed by the nudged elastic band (NEB) calculations. Among the Li-ion pathways observed in AIMD simulations, three of them were selected from the 2D defect layer (Fig. 5d) to calculate the Li-ion migration energy profiles. The Li-ion migration barrier was found barely affected by the local A-site configurations in the adjacent LLTO layers (Supplementary Note 1 and Supplementary Fig. 11), but dependent on the configuration of $\mathrm{Ti}, \mathrm{Li}$, and vacancies within the defect layer. The ground-state configuration of the defect layer from our calculations exhibits a chain-like $\mathrm{Ti}$ configuration (Fig. 5d), which is similar to that in the (202) plane of $\beta-\mathrm{Li}_{2} \mathrm{TiO}_{3}$ (Supplementary Fig. 12). Li-ion migration across this $\mathrm{Ti}$ chain exhibits a high barrier of $\sim 1.2 \mathrm{eV}$ (path 2 in Fig. $5 \mathrm{~d}$, e), and the migration besides the Ti chain shows a barrier of $\sim 0.8 \mathrm{eV}$ (paths 1 and 3 in Fig. 5d, e). The Li-ion diffusion in the $2 \mathrm{D}$ defect layer was compared with that in $\beta-\mathrm{Li}_{2} \mathrm{TiO}_{3}$, where similar pathways as those indicated in Fig. 5d (Supplementary Fig. 12) were selected for study. It was found that the $2 \mathrm{D}$ defect layer exhibited much higher energy barriers $(0.8-1.2 \mathrm{eV})$ than $\beta-\mathrm{Li}_{2} \mathrm{TiO}_{3}$ $(0.49-0.53 \mathrm{eV}$, consistent with the experimental and theoretical studies in literature $\left.{ }^{53,54}\right)$. As a result, Li-ion migration within the $2 \mathrm{D}$ defect is likely to be slower than that in $\beta-\mathrm{Li}_{2} \mathrm{TiO}_{3}$, a material with only $2.43 \times 10^{-12} \mathrm{~S} \mathrm{~cm}^{-1}$ conductivity ${ }^{52}$ (Note: this by no means suggests that the "SALT conductivity" acquired by fitting the Nyquist plot of LLTO should supposedly be lower than $2.43 \times$ $10^{-12} \mathrm{~S} \mathrm{~cm}^{-1}$. The conductivity obtained from the Nyquist plot of LLTO is a fundamentally different physical quantity from what is being estimated here. The details are explained in Supplementary Note 2).

Although such an extremely slow Li-ion migration appeared to happen only in the single-atom-thick defect layers, the SALTs formed by these defects have very profound influences on the overall ionic transport. It should be noted that the LLTO atomic planes adjacent to the $2 \mathrm{D}$ defect always became the La-rich A-site layers (Fig. 1a-d), which were known to be Li-ion-blocking?. Therefore, Li-ion diffusion across the $2 \mathrm{D}$ defect would be even more difficult than that within the defect layer. Since the latter was already slower than $2.43 \times 10^{-12} \mathrm{~S} \mathrm{~cm}^{-1}$, ionic transport through the defect layer should be forbidden; in fact, such a transport behavior, according to our AIMD simulation results described above, was indeed absent even at $2000 \mathrm{~K}$. When these Li-ion-blocking 2D defects form SALTs to enclose 3D volumes,
$\mathrm{Li}^{+}$would not be able to enter or escape the regions inside. The enclosed volumes are thus isolated from the rest of the material, and can hardly participate in the overall ionic transport. Presently it is difficult to tell whether the SALTs or the grain boundaries ${ }^{3,6}$ are more severely impeding the ionic transport, because the reliable simulation of grain boundaries is challenging as reflected by the rareness of such studies ${ }^{14,15,28,29}$. Nevertheless, the Li-ionblocking SALTs are ubiquitously present like grain boundaries, and the volumes they isolated are also considerable (the grain size is $2-4 \mu \mathrm{m}$, while the volume enclosed by the individual SALT varies between $15 \mathrm{~nm}$ and $1 \mu \mathrm{m}$; besides, many grains possess multiple SALTs, as shown in Supplementary Figs. 4 and 5). As a result, these features should cause massive degradation to the total ionic conductivity too. In order to evaluate the significance of such effect, we estimated the average volume fraction that was isolated by SALTs in each grain. The estimation covers all the grains that had been selected (randomly) for observation in this study, including those without SALTs; for SALT-free grains, the isolated volume fraction was counted as $0 \%$. In this way, it was estimated that averagely $15.7 \mathrm{vol} \%$ of LLTO is isolated by SALTs. As demonstrated above, these isolated volumes cannot participate in the overall ionic transport, so in practice they are nonconductive, like pores in the ceramic. Consequently, although the LLTO pellet used in the present study (relative density 96.3\%) only carries $3.7 \mathrm{vol} \%$ of pores, the presence of SALTs is equivalent to making an additional 15.1 vol\% $(=96.3 \% \times 15.7 \%)$ of the ceramic into non-conductive pores. For oxide solid electrolytes, when the porosity increases from $3.7 \%$ to $18.8 \%(=3.7 \%+$ $15.1 \%)$, the conductivity would decrease by $1-2$ orders of magnitude, regardless of the material system ${ }^{55-59}$. Supposedly, SALTs are causing similar conductivity degradation, so they should no longer be left unattended. The potential approaches to eliminate SALTs are yet to be explored. However, our estimated formation energies (Supplementary Note 3) suggest that the emergence of SALTs might be favored by the loss of Li and/or O during synthesis, which frequently happens due to the high sintering temperature of $\mathrm{LLTO}^{34}$. If future studies can develop corresponding strategies to minimize the population of SALTs, further improvement in total ionic conductivity can be expected. Clearly, non-periodic features beyond point defects and grain boundaries could be vital in both comprehending the ionic transport mechanism and optimizing the materials performances. Therefore, they must be thoroughly investigated in all of the important solid electrolyte systems.

\section{Discussion}

In summary, we discovered an additional type of non-periodic feature that could greatly influence ionic transport, and a term "single-atom-layer trap" (SALT) was coined to describe this phenomenon. SALTs are closed loops formed by single-atom-layer 2D defects. Although this feature has never been discussed in previous mechanistic studies, our atomic-resolution STEM observation spotted numerous SALTs in a prototype solid electrolyte $\mathrm{Li}_{0.33} \mathrm{La}_{0.56} \mathrm{TiO}_{3}$. Based on the experimentally determined defect structure, AIMD simulations suggested that $\mathrm{Li}$ ions are impossible to migrate across the $2 \mathrm{D}$ defects constituting SALTs. Consequently, the volumes enclosed by SALTs cannot participate in the overall ionic transport. Although further study is needed to compare the effect of SALTs and that of grain boundaries ${ }^{14,15,28,29}$, the large population of SALTs entails that these Li-ion-blocking features must be causing considerable degradation to the total conductivity. This discovery demonstrated that non-periodic features apart from the point defects and grain boundaries could severely impact the Li-ion migration too. In order to fully comprehend the ionic 
transport mechanisms, similar study should be performed to other solid electrolyte systems.

\begin{abstract}
Methods
Materials and macroscopic characterizations. The LLTO ceramics were prepared through a sol-gel approach. Stoichiometric amount of $\mathrm{LiNO}_{3}$ and $\mathrm{La}$ $\left(\mathrm{NO}_{3}\right)_{3} \cdot 6 \mathrm{H}_{2} \mathrm{O}$ were dissolved in ethylene glycol monomenthyl ether, and then mixed with tetrabutyl titanate and acetylacetone. After drying at $70^{\circ} \mathrm{C}$, the gel was calcined at $900^{\circ} \mathrm{C}$ for $6 \mathrm{~h}$. The calcined powder was then ball milled for $12 \mathrm{~h}$, pressed into a pellet, sintered at $1350^{\circ} \mathrm{C}$ for $6 \mathrm{~h}$, and finally annealed at $800{ }^{\circ} \mathrm{C}$ for three days to form the dense LLTO ceramic with the tetragonal structure. To compensate for Li loss at high temperatures, the pellets were buried in powders with the same composition during the heat treatment described above. The density of the sintered pellet was determined using Archimedes' principle. The crystal structure, stoichiometry, and ionic conductivity of the pellets were confirmed by Xray diffraction, inductively coupled plasma spectroscopy, and electrochemical impedance spectroscopy, respectively. The ionic conductivity was also measured by the DC method using the experimental configuration reported by Inaguma et al. ${ }^{35}$.
\end{abstract}

Electron microscopy. TEM specimens were prepared by mechanically thinning the LLTO ceramic followed by Ar-ion milling with liquid nitrogen cooling at around $-100^{\circ} \mathrm{C}$. The ion milling was performed at $3 \mathrm{kV}$ and $4 \mathrm{~mA}$ at first. Upon perforation, the specimen was milled at $1.5 \mathrm{kV}$ and $3 \mathrm{~mA}$ to remove the surface amorphous layer. The ion-milled specimens were stored in $10^{-5}$ torr vacuum until electron microscopy observation. The STEM/EELS study was conducted on an aberration-corrected FEI Titan Themis TEM/STEM equipped with a Gatan Image Filter Quantum-965. To avoid possible electron beam damage, the microscope was operated at $200 \mathrm{kV}$ with dose rates below $12 \mathrm{e}^{-} \AA^{-2} \mathrm{~s}^{-1}$. The STEM images presented here were Fourier-filtered to minimize the contrast noise, and such processing did not introduce any artifact that may alter our conclusions. The simulation of STEM images was performed using the QSTEM simulation package. The strain maps were calculated from the STEM image using the commercial GPA software of HREM Research ${ }^{47}$. The volume fraction isolated by SALTs in each grain was determined using the software ImageJ. The EELS data were acquired in STEM mode with a $5 \mathrm{~mm}$ aperture and an energy dispersion of $0.1 \mathrm{eV}$ per channel.

First principles computation. All of the DFT calculations were performed using the projector augmented-wave (PAW) ${ }^{60}$ approach using Perdew-Burke-Ernzerhof $(\mathrm{PBE})^{61}$ generalized-gradient approximation (GGA) implemented in the Vienna ab initio simulation package (VASP) ${ }^{62}$. All structure optimization calculations were performed using the convergence parameters set of the Materials Project ${ }^{63}$. The defect layer was modelled in a supercell consisting of two slabs of LLTO and two defect layers using the periodic boundary condition (CIF file attached as Supplementary Data 1). From the disordered structure of LLTO and the defect layer from experiments, the atomistic configuration of $\mathrm{Li}, \mathrm{La}$, and $\mathrm{Ti}$ in the supercell models was generated in a two-step process by comparing and ranking distinctive configurations using the scheme as in our previous work $\mathrm{k}^{64}$ according to electrostatic energies and DFT energies: (1) the atomistic configuration with the lowest GGA energy was selected from the DFT calculations on 100 distinctive configurations, which were obtained according to the minimum electrostatic energy out of 10000 symmetrically distinctive configurations using pymatgen ${ }^{65}$; $(2)$ the same process was repeated for ordering the atomistic configuration in the $2 \mathrm{D}$ defect layers to obtain a low-energy structure model of the defect for further calculations. The NEB calculations were performed with the supercell model of $11.83 \AA$ x $11.75 \AA \mathrm{x}$ $31.84 \AA$ containing 378 atoms (detailed structure described in the CIF file attached as Supplementary Data 1). The paths of the Li-ion migration in the $2 \mathrm{D}$ defect were chosen from those observed in the AIMD simulation. For $\beta-\mathrm{Li}_{2} \mathrm{TiO}_{3}$, the possible migration pathways similar to those in the defect layer were considered. NEB calculations were performed using nine images linearly interpolated from fully relaxed initial and final structure, and were converged within $0.01 \mathrm{eV} \AA^{-1}$. To accomplish longer simulation time with reasonable computational cost, we conducted AIMD simulations on a smaller supercell model of $11.83 \AA$ x $11.75 \AA$ x $16.62 \AA$ containing 202 atoms, in which the La-poor layer in the LLTO slab was removed. The AIMD simulation, which was non-spin polarized using single $\Gamma$-centered $k$-point, was performed at $2000 \mathrm{~K}$ with a time-step of $2 \mathrm{fs}$ in NVT using a Nosé-Hoover thermostat ${ }^{66}$ for a total time of $80 \mathrm{ps}$. Since $\mathrm{Li}$ ions in the $2 \mathrm{D}$ defect were observed to migrate always within itself in our simulations, the Li-ion diffusivity of the $2 \mathrm{D}$ defect was evaluated from the displacements of all $\mathrm{Li}$ ions in this particular atomic layer using the scheme established in the previous study ${ }^{67}$.

\section{Data availability}

The authors declare that all relevant data are included in the paper and its Supplementary Information file. Additional data are available from the corresponding authors upon reasonable request.

Received: 10 August 2019; Accepted: 18 March 2020; Published online: 14 April 2020

\section{References}

1. Janek, J. \& Zeier, W. G. A solid future for battery development. Nat. Energy 1 16141 (2016).

2. Manthiram, A., Yu, X. \& Wang, S. Lithium battery chemistries enabled by solid-state electrolytes. Nat. Rev. Mater. 2, 16103 (2017)

3. Bachman, J. C. et al. Inorganic solid-state electrolytes for lithium batteries: mechanisms and properties governing ion conduction. Chem. Rev. 116, 140-162 (2016).

4. Takada, K. Progress in solid electrolytes toward realizing solid-state lithium batteries. J. Power Sources 394, 74-85 (2018).

5. Wang, Y. et al. Design principles for solid-state lithium superionic conductors. Nat. Mater. 14, 1026-1031 (2015).

6. $\mathrm{Ma}, \mathrm{C}$. et al. Atomic-scale origin of the large grain-boundary resistance in perovskite Li-ion-conducting solid electrolytes. Energy Environ. Sci. 7, 1638 (2014).

7. Ma, C. et al. Mesoscopic framework enables facile ionic transport in solid electrolytes for Li batteries. Adv. Energy Mater. 6, 1600053 (2016).

8. Miao, J., Ercius, P. \& Billinge, S. J. L. Atomic electron tomography: 3D structures without crystals. Science 353, aaf2157 (2016).

9. Kinoshita, C. Characteristics of defects in ceramic materials. Radiat. Eff. Defect. S. 148, 63 (1999).

10. Billinge, S. J. L. \& Levin, I. The problem with determining atomic structure at the nanoscale. Science 316, 561-565 (2007).

11. Asano, T. et al. Solid halide electrolytes with high lithium-ion conductivity for application in $4 \mathrm{~V}$ class bulk-type all-solid-state batteries. Adv. Mater. 30, 1803075 (2018).

12. Liu, Z. et al. Anomalous high ionic conductivity of nanoporous beta- $\mathrm{Li}_{3} \mathrm{PS}_{4}$. J. Am. Chem. Soc. 135, 975-978 (2013).

13. Rangasamy, E. et al. A high conductivity oxide-sulfide composite lithium superionic conductor. J. Mater. Chem. A 2, 4111-4116 (2014).

14. Dawson, J. A., Canepa, P., Famprikis, T., Masquelier, C. \& Islam, M. S. Atomic-scale influence of grain boundaries on Li-ion conduction in solid electrolytes for all-solid-state batteries. J. Am. Chem. Soc. 140, 362-368 (2018).

15. Yu, S. \& Siegel, D. J. Grain boundary contributions to Li-ion transport in the solid electrolyte $\mathrm{Li}_{7} \mathrm{La}_{3} \mathrm{Zr}_{2} \mathrm{O}_{12}$ (LLZO). Chem. Mater. 29, 9639-9647 (2017).

16. Canepa, P. et al. Particle morphology and lithium segregation to surfaces of the $\mathrm{Li}_{7} \mathrm{La}_{3} \mathrm{Zr}_{2} \mathrm{O}_{12}$ solid electrolyte. Chem. Mater. 30, 3019-3027 (2018).

17. Deng, Y. et al. Structural and mechanistic insights into fast lithium-ion conduction in $\mathrm{Li}_{4} \mathrm{SiO}_{4}-\mathrm{Li}_{3} \mathrm{PO}_{4}$ solid electrolytes. J. Am. Chem. Soc. 137, 9136-9145 (2015)

18. Panchmatia, P. M., Armstrong, A. R., Bruce, P. G. \& Islam, M. S. Lithium-ion diffusion mechanisms in the battery anode material $\mathrm{Li}_{1+x} \mathrm{~V}_{1-x} \mathrm{O}_{2}$. Phys. Chem. Chem. Phys. 16, 21114-21118 (2014).

19. Jalem, R. et al. Concerted migration mechanism in the Li ion dynamics of garnet-type $\mathrm{Li}_{7} \mathrm{La}_{3} \mathrm{Zr}_{2} \mathrm{O}_{12}$. Chem. Mater. 25, 425-430 (2013).

20. Meier, K., Laino, T. \& Curioni, A. Solid-state electrolytes: revealing the mechanisms of Li-ion conduction in tetragonal and cubic LLZO by firstprinciples calculations. J. Phys. Chem. C. 118, 6668-6679 (2014).

21. He, X. F., Zhu, Y. Z. \& Mo, Y. F. Origin of fast ion diffusion in super-ionic conductors. Nat. Commun. 8, 15893 (2017).

22. Xiong, $\mathrm{S}$. et al. Computation-guided design of $\mathrm{LiTaSiO}_{5}$, a new lithium ionic conductor with sphene structure. Adv. Energy Mater. 9, 1803821 (2019).

23. Dawson, J. A. et al. Elucidating lithium-ion and proton dynamics in antiperovskite solid electrolytes. Energy Environ. Sci. 11, 2993-3002 (2018).

24. Liu, $\mathrm{X}$. et al. Elucidating the mobility of $\mathrm{H}^{+}$and $\mathrm{Li}^{+}$ions in $\left(\mathrm{Li}_{6.25-x} \mathrm{H}_{x} \mathrm{Al}_{0.25}\right)$ $\mathrm{La}_{3} \mathrm{Zr}_{2} \mathrm{O}_{12}$ via correlative neutron and electron spectroscopy. Energy Environ. Sci. 12, 945-951 (2019).

25. Dawson, J. A., Chen, H. R. \& Islam, M. S. Composition screening of lithiumand sodium-rich anti-perovskites for fast-conducting solid electrolytes. J. Phys. Chem. C. 122, 23978-23984 (2018).

26. Deng, Y. et al. Enhancing the lithium ion conductivity in lithium superionic conductor (LISICON) solid electrolytes through a mixed polyanion effect. ACS Appl. Mater. Interfaces 9, 7050-7058 (2017).

27. Famprikis, T. et al. A new superionic plastic polymorph of the $\mathrm{Na}^{+}$conductor $\mathrm{Na}_{3} \mathrm{PS}_{4}$. ACS Mater. Lett. 1, 641-646 (2019).

28. Dawson, J. A. et al. Toward understanding the different influences of grain boundaries on ion transport in sulfide and oxide solid electrolytes. Chem. Mater. 31, 5296-5304 (2019).

29. Yu, S. \& Siegel, D. J. Grain boundary softening: a potential mechanism for lithium metal penetration through stiff solid electrolytes. ACS Appl. Mater. Interfaces 10, 38151-38158 (2018).

30. Famprikis, T., Canepa, P., Dawson, J. A., Islam, M. S. \& Masquelier, C. Fundamentals of inorganic solid-state electrolytes for batteries. Nat. Mater. 18, 1278-1291 (2019).

31. Pelleg, J. in Mechanical Properties of Ceramics 173-279 (Springer International Publishing, 2014).

32. Ren, Y. et al. Oxide electrolytes for lithium batteries. J. Am. Ceram. Soc. 98 , 3603-3623 (2015) 
33. Stramare, S., Thangadurai, V. \& Weppner, W. Lithium lanthanum titanates: a review. Chem. Mater. 15, 3974-3990 (2003).

34. Bohnke, $\mathrm{O}$. The fast lithium-ion conducting oxides $\mathrm{Li}_{3 x} \mathrm{La}_{2 / 3-x} \mathrm{TiO}_{3}$ from fundamentals to application. Solid State Ion. 179, 9-15 (2008).

35. Inaguma, Y. et al. High ionic conductivity in lithium lanthanum titanate. Solid State Commun. 86, 689-693 (1993).

36. Pennycook, S. J. Z-contrast transmission electron microscopy: direct atomic imaging of materials. Annu. Rev. Mater. Sci. 22, 171-195 (1992).

37. Egerton, R. F. Electron energy-loss spectroscopy in the TEM. Rep. Prog. Phys. 72, 016502 (2009).

38. Leapman, R. D. \& Hunt, J. A. Comparison of detection limits for EELS and EDXS. Microsc. Microanal. Microstruct. 2, 231-244 (1991).

39. Saitoh, M. et al. Systematic analysis of electron energy-loss near-edge structures in Li-ion battery materials. Phys. Chem. Chem. Phys. 20, 25052-25061 (2018).

40. Cheng, Y. Q. et al. An integrated approach for structural characterization of complex solid state electrolytes: the case of lithium lanthanum titanate. J. Mater. Chem. A 2, 2418-2426 (2014).

41. Wang, Z. L., Yin, J. S. \& Jiang, Y. D. EELS analysis of cation valence states and oxygen vacancies in magnetic oxides. Micron 31, 571-580 (2000).

42. Stoyanov, E., Langenhorst, F. \& Steinle-Neumann, G. The effect of valence state and site geometry on Ti- $L_{3,2}$ and O-K electron energy-loss spectra of $\mathrm{Ti}_{x} \mathrm{O}_{y}$ phases. Am. Mineral. 92, 577-586 (2007).

43. Laumann, A., Fehr, K. T., Wachsmann, M., Holzapfel, M. \& Iversen, B. B. Metastable formation of low temperature cubic $\mathrm{Li}_{2} \mathrm{TiO}_{3}$ under hydrothermal conditions-Its stability and structural properties. Solid State Ion. 181, 1525-1529 (2010).

44. Zhu, Y. Y., Song, C. Y., Minor, A. M. \& Wang, H. Y. C $_{\mathrm{s}}$-corrected scanning transmission electron microscopy investigation of dislocation core configurations at a $\mathrm{SrTiO}_{3} / \mathrm{MgO}$ heterogeneous interface. Microsc. Microanal. 19, 706-715 (2013).

45. Chern, G. \& Cheng, C. Interface matching in oxides of rocksalt/rocksalt(001) and rocksalt/perovskite(001). J. Vac. Sci. Technol. A 17, 1097-1102 (1999).

46. Findlay, S. D. et al. Dynamics of annular bright field imaging in scanning transmission electron microscopy. Ultramicroscopy 110, 903-923 (2010).

47. Hÿtch, M. J., Snoeck, E. \& Kilaas, R. Quantitative measurement of displacement and strain fields from HREM micrographs. Ultramicroscopy 74, 131-146 (1998).

48. Li, F. et al. Atomically intimate contact between solid electrolytes and electrodes for Li batteries. Matter 1, 1001-1016 (2019).

49. Catti, M. Ion mobility pathways of the $\mathrm{Li}^{+}$conductor $\mathrm{Li}_{0.125} \mathrm{La}_{0.625} \mathrm{TiO}_{3}$ by ab initio simulations. I. Phys. Chem. C. 112, 11068-11074 (2008).

50. Sommariva, M. \& Catti, M. Neutron diffraction study of quenched $\mathrm{Li}_{0.3} \mathrm{La}_{0.567} \mathrm{TiO}_{3}$ lithium ion conducting perovskite. Chem. Mater. 18, 2411-2417 (2006).

51. Monchak, M. et al. Monoclinic $\beta-\mathrm{Li}_{2} \mathrm{TiO}_{3}$ : neutron diffraction study and estimation of Li diffusion pathways. Solid State Sci. 61, 161-166 (2016).

52. Wu, X. W., Wen, Z. Y., Xu, X. G. \& Han, J. D. Synthesis and ionic conductivity of Mg-doped $\mathrm{Li}_{2} \mathrm{TiO}_{3}$. Solid State Ion. 179, 1779-1782 (2008).

53. Ruprecht, B., Wilkening, M., Uecker, R. \& Heitjans, P. Extremely slow Li ion dynamics in monoclinic $\mathrm{Li}_{2} \mathrm{TiO}_{3}$-probing macroscopic jump diffusion via ${ }^{7} \mathrm{Li}$ NMR stimulated echoes. Phys. Chem. Chem. Phys. 14, 11974-11980 (2012).

54. Islam, M. M. \& Bredow, T. Lithium diffusion pathways in beta- $\mathrm{Li}_{2} \mathrm{TiO}_{3}$ : a theoretical study. J. Phys. Chem. C. 120, 7061-7066 (2016)

55. Chen, C. H. et al. Stable lithium-ion conducting perovskite lithium-strontiumtantalum-zirconium-oxide system. Solid State Ion. 167, 263-272 (2004).

56. Ban, C. W. \& Choi, G. M. The effect of sintering on the grain boundary conductivity of lithium lanthanum titanates. Solid State Ion. 140, 285-292 (2001).

57. Huang, $\mathrm{X}$. et al. Preparation of dense Ta-LLZO/MgO composite Li-ion solid electrolyte: Sintering, microstructure, performance and the role of $\mathrm{MgO}$. J. Energy Chem. 39, 8-16 (2019).

58. Hallopeau, L. et al. Microwave-assisted reactive sintering and lithium ion conductivity of $\mathrm{Li}_{1.3} \mathrm{Al}_{0.3} \mathrm{Ti}_{1.7}\left(\mathrm{PO}_{4}\right)_{3}$ solid electrolyte. J. Power Sources 378, 48-52 (2018)

59. Wolfenstine, J., Rangasamy, E., Allen, J. L. \& Sakamoto, J. High conductivity of dense tetragonal $\mathrm{Li}_{7} \mathrm{La}_{3} \mathrm{Zr}_{2} \mathrm{O}_{12}$. J. Power Sources 208, 193-196 (2012).

60. Blöchl, P. E. Projector augmented-wave method. Phys. Rev. B 50, 17953-17979 (1994).

61. Perdew, J. P., Emzerhof, M. \& Burke, K. Rationale for mixing exact exchange with density functional approximations. J. Chem. Phys. 105, 9982-9985 (1996).

62. Kresse, G. \& Hafner, J. Ab initio molecular dynamics for liquid metals. Phys. Rev. B 47, 558-561 (1993).

63. Jain, A. et al. A high-throughput infrastructure for density functional theory calculations. Comp. Mater. Sci. 50, 2295-2310 (2011)
64. Bai, Q., He, X., Zhu, Y. \& Mo, Y. First-principles study of oxyhydride H-ion conductors: toward facile anion conduction in oxide-based materials. ACS Appl. Energy Mater. 1, 1626-1634 (2018).

65. Ong, S. P. et al. Python Materials Genomics (pymatgen): a robust, opensource python library for materials analysis. Comp. Mater. Sci. 68, 314-319 (2013).

66. Nose, S. Constant temperature molecular-dynamics methods. Prog. Theor Phys. Supp 103, 1-46 (1991).

67. He, X., Zhu, Y., Epstein, A. \& Mo, Y. Statistical variances of diffusional properties from ab initio molecular dynamics simulations. npj Comput. Mater. 4, 18 (2018)

\section{Acknowledgements}

C.M. acknowledges the financial support from the National Key R\&D Program of China (2018YFA0209600, 2017YFA0208300), the National Natural Science Foundation of China (51802302), the Fundamental Research Funds for the Central Universities (WK2060190085, WK2340000076). J.L. acknowledges the financial support from National Key R\&D Program of China (2017YFA0700104) and National Natural Science Foundation of China (51761165012). C.-W.N. acknowledges the financial support from Basic Science Center Program of National Natural Science Foundation of China (51788104). The work in Ames Laboratory was supported by Laboratory Directed Research and Development funds and by the U.S. Department of Energy, Office of Science, Basic Energy Sciences, Materials Science and Engineering Division (L.Z.). Ames Laboratory is operated for the U.S. Department of Energy by Iowa State University under Contract No. DE-AC02-07CH11358. Y.M. acknowledges the financial support from National Science Foundation under award No. 1550423 and the computational facilities from the University of Maryland supercomputing resources, the Maryland Advanced Research Computing Center (MARCC).

\section{Author contributions}

Y.M. and C.M. planned and supervised the research. T.L. synthesized the LLTO ceramics T.L. and X.W. performed the macroscopic characterizations. L.Z. aligned the transmission electron microscope and assisted in data collection. F.Z. and C.M. performed electron microscopy observation and analyzed the electron microscopy data. The electron microscopy study performed by F.Z. was directed by C.M. and J.L. Z.G. collected the electron microscopy data shown in Supplementary Fig. 4. M.S.I. performed the calculations as directed by Y.M. F.Z. drafted the manuscript. C.M., Y.M. and C.-W.N. revised the manuscript drafted by F.Z. All authors discussed the results and the manuscript.

\section{Competing interests}

The authors declare no competing interests.

\section{Additional information}

Supplementary information is available for this paper at https://doi.org/10.1038/s41467 020-15544-x.

Correspondence and requests for materials should be addressed to Y.M. or C.M.

Peer review information Nature Communications thanks Theodosios Famprikis and the other, anonymous, reviewer(s) for their contribution to the peer review of this work

Reprints and permission information is available at http://www.nature.com/reprints

Publisher's note Springer Nature remains neutral with regard to jurisdictional claims in published maps and institutional affiliations.

Open Access This article is licensed under a Creative Commons Attribution 4.0 International License, which permits use, sharing, adaptation, distribution and reproduction in any medium or format, as long as you give appropriate credit to the original author(s) and the source, provide a link to the Creative Commons license, and indicate if changes were made. The images or other third party material in this article are included in the article's Creative Commons license, unless indicated otherwise in a credit line to the material. If material is not included in the article's Creative Commons license and your intended use is not permitted by statutory regulation or exceeds the permitted use, you will need to obtain permission directly from the copyright holder. To view a copy of this license, visit http://creativecommons.org/ licenses/by/4.0/

(C) The Author(s) 2020 